\section{Trasplante hepático exitoso en un paciente portador del Virus de la Inmunodeficiencia Humana (VIH)}

\author{
JUAN FRANCISCO GUERRA ${ }^{1,2}$, ANDRÉS TRONCOSO ${ }^{2}$, \\ MARÍA ELENA CEBALLOS ${ }^{3}$, MARCO ARRESE ${ }^{1,4}$, \\ FRANCISCO BARRERA ${ }^{1,4}$, BLANCA NORERO ${ }^{1,4}$, \\ ALEJANDRO SOZA ${ }^{1,4}$, VIOLETA RIVAS ${ }^{4}$, RODRIGO WOLFF ${ }^{1,4}$, \\ ALEJANDRA ARIAS ${ }^{1,4}$, ALEJANDRA CANCINO ${ }^{1,4}$, \\ JAVIERA TORRES ${ }^{5}$, EDUARDO BRICEÑO ${ }^{1,2}$, NICOLÁS JARUFE ${ }^{1,2}$, \\ JORGE MARTÍNEZ ${ }^{1,2}$, CARLOS BENÍTEZ ${ }^{1,4}$
}

\section{Liver transplantation in a patient with human immunodeficiency virus (HIV). Case report}

Before the advent of highly active antiretroviral therapy (HAART), patients infected with human immunodeficiency virus (HIV) were considered as having an absolute contraindication for liver transplantation (LT). Considering the increased life expectancy in HIV positive patients under HAART and the improvements in the management of graft recipients, these patients are now suitable for carrying out transplants in selected cases. We report a 26 years old HIV positive male who developed acute liver failure possibly caused by drug induced liver injury who underwent a successful liver transplantation.

(Rev Med Chile 2018; 146: 1215-1219)

Key words: Antiretroviral Therappy, Highly Active; Chemical and Drug Induced; HIV; Liver Failure, Acute; Liver Injury; Liver Transplantation.
'Programa Trasplante Hepático.

Hospital Clínico Pontificia

Universidad Católica de Chile.

Santiago, Chile.

2Departamento de Cirugía Digestiva. Pontificia Universidad

Católica de Chile. Santiago, Chile.

${ }^{3}$ Departamento de Enfermedades

Infecciosas del Adulto. Pontificia

Universidad Católica de Chile.

Santiago, Chile.

${ }^{4}$ Departamento de

Gastroenterología. Pontificia

Universidad Católica de Chile.

Santiago, Chile.

${ }^{5}$ Departamento de Anatomía

Patológica. Pontificia Universidad

Católica de Chile. Santiago, Chile.

Los autores declaran no tener conflictos de intereses.

Trabjo no recibio finaciamiento.

Recibido el 2 de marzo de 2018, aceptado el 14 septiembre de 2018.

Correspondencia a:

Dr. Carlos Benítez Gajardo Diagonal Paraguay 362.

40 Piso. Departamento de Gastroenterología. Santiago,

Chile.

cbenitez@uc.cl
E 1 trasplante hepático $(\mathrm{TH})$ es la terapia de elección para la insuficiencia hepática en su forma aguda o crónica, con supervivencias del injerto y el paciente de $89,7 \%$ y $90 \%$ al año y $71 \%$ y $72 \%$ a 5 años respectivamente ${ }^{1}$. Previo a la aparición de la Terapia Antirretroviral Efectiva (TARV) el TH se consideraba contraindicado en portadores del virus de la inmunodeficiencia humana (VIH) debido a los limitados resultados obtenidos por complicaciones derivadas de la infección viral y los inmunosupresores utilizados para trasplante $e^{2,3}$. No obstante, la disponibilidad de TARV permitió que la mortalidad relacionada a la infección por VIH disminuyera drásticamente 4 . Simultáneamente, se ha observado un aumento de enfermedades no asociadas al VIH, como las cardiovasculares o hepáticas, las cuáles se han convertido en importantes causas de morbimortalidad en este grupo de pacientes ${ }^{3}$.

Considerando el aumento de la expectativa de vida en pacientes con infección por VIH asociado a la utilización de TARV, el mayor desarrollo de la medicina de trasplantes, mejores cuidados perioperatorios y resultados quirúrgicos, el TH ha llegado a ser una alternativa en pacientes portadores de VIH con insuficiencia hepática, toda vez que esta infección se encuentre controlada ${ }^{3}$.

Presentamos el caso de un paciente portador de VIH sometido exitosamente a un TH. 


\section{Caso clínico}

Paciente masculino de 26 años con antecedente de infección por VIH de 5 años de evolución en TARV con abacavir/lamivudina/dolutegravir (con alelo HLA-B ${ }^{\star} 5701$ negativo), y con adecuado control inmunovirológico. Además con asma bronquial en tratamiento, sífilis latente tardía, infección por virus de hepatitis $\mathrm{B}(\mathrm{VHB})$ resuelta $(\mathrm{HBc}$ total $(+)$, HBsAG $(-))$ y resistencia a la insulina en tratamiento con metformina. Es hospitalizado por cuadro de 2 semanas de evolución caracterizado por compromiso del estado general, ictericia y coluria. Al ingreso destaca GOT 839 U/L, GPT $1135 \mathrm{U} / \mathrm{L}$, bilirrubina total de $18,6 \mathrm{mg} /$ dL e INR 1,7 .

El estudio etiológico fue negativo incluyendo IgG total $(1.630 \mathrm{mg} / \mathrm{dL}$, valor normal para nuestro laboratorio), anticuerpos antinucleares (ANA), anti músculo liso (ASMA), anti mitocondriales (AMA), anticuerpos anti LKM-1, IgM para virus hepatitis A y E, antígeno de superficie virus hepatitis $\mathrm{B}(\mathrm{HBsAg})$, serología para virus hepatitis C, PCR para virus Epstein Barr negativas y ceruloplasmina $(32 \mathrm{mg} / \mathrm{dL})$. La resonancia nuclear magnética no mostró evidencia de enfermedad hepática pre-existente, con vasculatura permeable. Se realizó una biopsia hepática percutánea con aguja de Menghini que mostró una hepatitis portal y lobulillar con necrosis en puente de hepatocitos sin depósitos de cobre ni otros elementos sugerentes de una etiología específica (Figura 1 a y b).

Previamente el paciente usó metformina por 48 meses. La TARV fue iniciada 8 meses previo al ingreso. Además, al interrogatorio se obtiene el antecedente de uso fentermina cuatro meses previo a su presentación. Se sospecha hepatoxicidad secundaria a fármacos (pese a la imposibilidad de determinar claramente la droga potencialmente responsable) o su sigla en inglés "DILI" (drug induced liver injury) y se decide suspender todos sus fármacos, incluída la TARV.

Se registró un deterioro progresivo de pruebas hepáticas (bilirrubina 39,3 mg/dL e INR hasta 2,4) asociado a encefalopatía grado 2 . La tomografía computada de cerebro fue normal. Se decide enlistar al paciente para trasplante por falla hepática fulminante considerando la presencia de tres criterios menores de la clasificación del King's College ${ }^{5}$ (bilirrubina $>17 \mathrm{mg} / \mathrm{dL}$, intervalo de encefalopatía desde ictericia más de 7 días y etiología desfavo- rable (DILI)). Dada la necesidad de encontrarse con cargas virales indetectables previo a un posible $\mathrm{TH}$, se inicia nueva terapia con tenofovir/emtricitabina/raltegravir. El paciente tuvo carga viral para VIH indetectable y recuento de linfocitos CD4+ de 427 por $\mathrm{mm}^{3}$ previo a trasplante.

Un día posterior al enlistamiento y 21 días posterior a su ingreso hospitalario, se realiza $\mathrm{TH}$ con técnica clásica (resección de vena cava) sin mayores incidentes (donante cadavérico, masculino, 19 años, muerte encefálica secundaria a TEC grave). Tiempo de isquemia fría $5 \mathrm{~h}$ y sangrado intraoperatorio de $1.000 \mathrm{cc}$. A las $24 \mathrm{~h}$ requirió reexploración en pabellón objetivándose un sangrado perianastomótico arterial, resuelto con sutura hemostática.

El paciente evolucionó estable iniciándose inmunosupresión estándar con tacrolimus, micofenolato y esteroides, destacando en su evolución un rechazo celular agudo moderado Banff 7 , manejado con bolos de metilprednisolona ( $1 \mathrm{~g} /$ día por 3 días) y ajuste de la terapia inmunosupresora.

El paciente evolucionó favorablemente siendo dado de alta 18 días post-trasplante, con excelente función del injerto, adecuados niveles de inmunosupresión y su TARV. El informe histopatológico del explante evidenció necrosis hepática submasiva sin elementos de especificidad (Figura $1 \mathrm{c} \mathrm{yd}$ ). El paciente se ha mantenido estable tras 12 meses de seguimiento.

\section{Discusión}

Antes de la aparición de TARV, la infección por VIH se consideraba una contraindicación absoluta para el trasplante de órganos sólidos ${ }^{2,6,7}$. El advenimiento de TARV contribuyó al replanteamiento del trasplante de órganos sólidos en estos pacientes dado el aumento de su expectativa de vida y el aumento en la prevalencia de insuficiencia hepática y renal.

Históricamente, los factores asociados a un mal pronóstico de pacientes $\mathrm{VIH}+$ sometidos a trasplante, incluían una inmunosupresión intensa (recuento bajo de linfocitos T CD4+ e inmunosupresión farmacológica), reducida expectativa de vida y aparición de infecciones oportunistas potencialmente graves. No obstante, estudios recientes ${ }^{7-9}$ han reportado que pacientes con recuento linfocitario CD 4+ estable mayor a 100 células/ 
Trasplante hepático en paciente portador de VIH - J. F. Guerra et al

$\mu \mathrm{L}$, carga viral indetectable y en TARV tienen tasas de mortalidad similar a pacientes no infectados 9 .

Actualmente, la terapia inmunosupresora post-trasplante en pacientes VIH no difiere de manera significativa respecto a la empleada en un paciente no VIH, pero deben ser monitorizados muy estrictamente ${ }^{4,6}$. Usualmente, esta terapia se basa en inhibidores de calcineurina (tacrolimus o ciclosporina), antimetabolitos como el micofe- nolato (que podría ser efectivo en reducir la carga viral de VIH a través de mecanismos virológicos e inmunológicos) ${ }^{10} \mathrm{y}$ esteroides, siendo el objetivo final de la terapia mantener un balance adecuado para minimizar la aparición de rechazo del injerto y evitar la aparición de infecciones oportunistas manteniendo el control farmacológico del VIH. En relación a la TARV, el uso de inhibidores de la proteasa se asocia a una gran disminución del
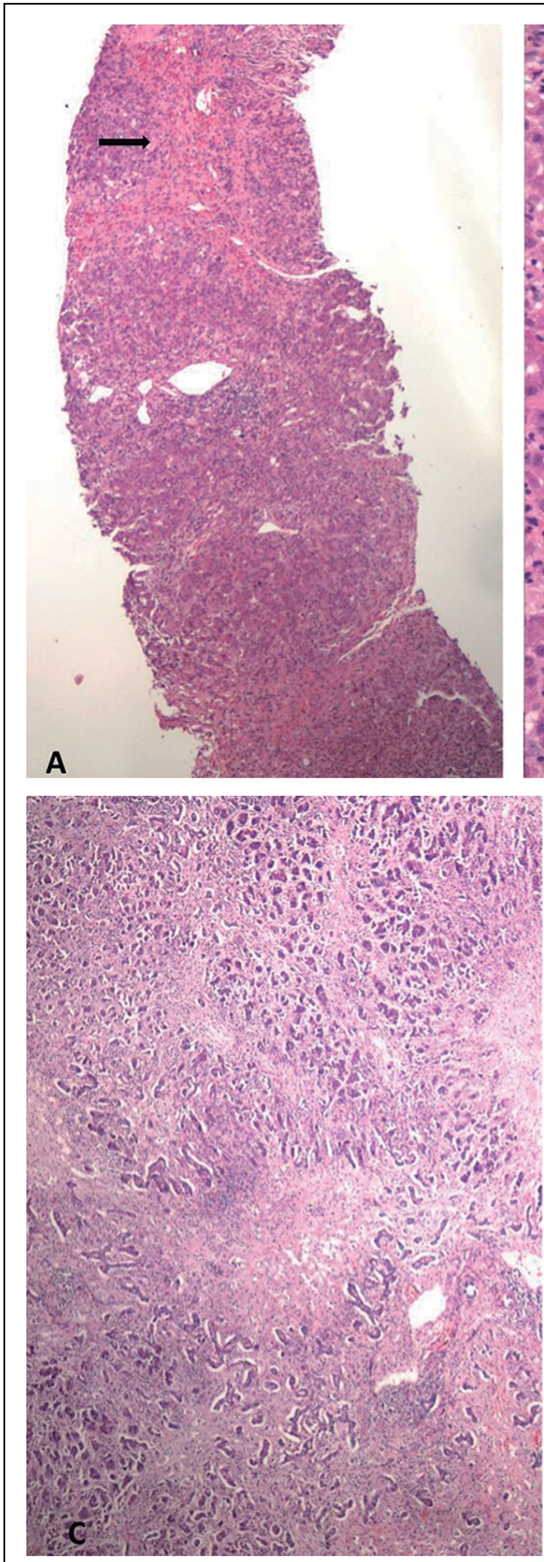
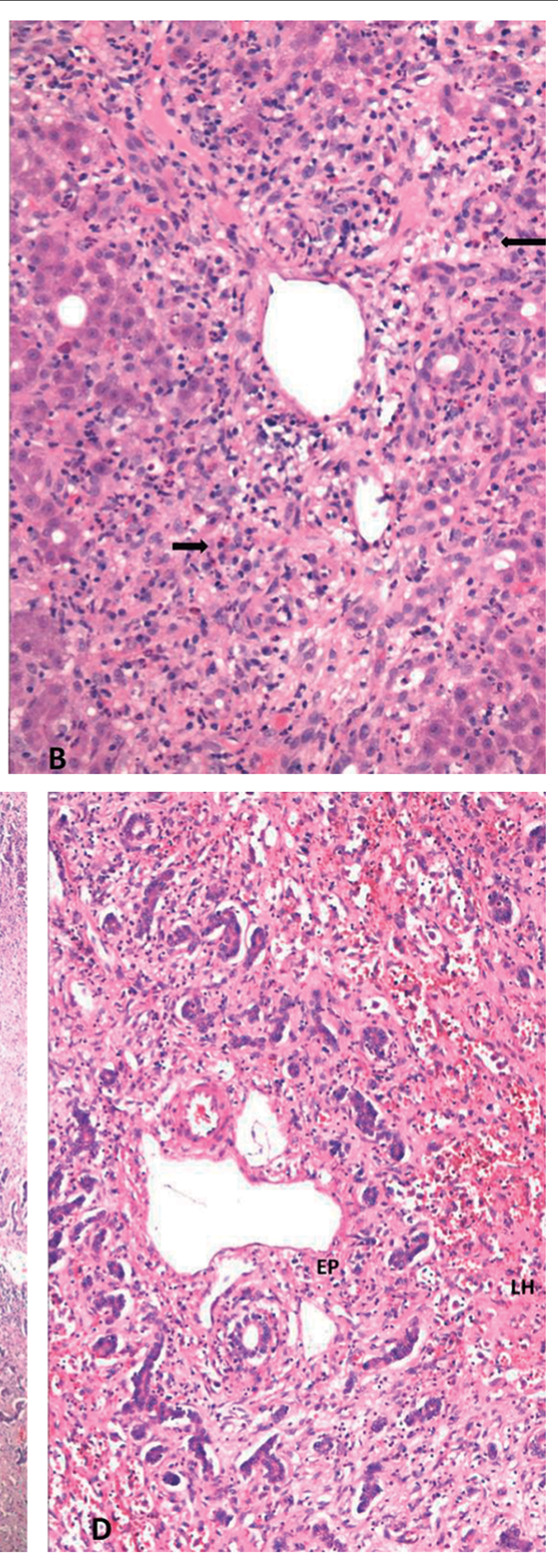

Figura 1. A. Tejido hepático con proceso inflamatorio portal y lobulillar, con necrosis en puente de hepatocitos (flecha); B. Espacio porta edematoso, con necrosis de hepatocitos de zona 1 e infiltrado inflamatorio en el que resaltan los eosinófilos (flechas), con aislados plasmocitos. C. Microfotografía del explante hepático. Extensa necrosis de hepatocitos. D. Espacio porta con leve infiltrado inflamatorio. Conducto biliar nativo indemne. Se reconoce extensa necrosis de hepatocitos del lobulillo hepático. 
metabolismo de los inhibidores de calcineurina debido a una inhibición del citocromo P450. Por otro lado, los inhibidores no nucleósidos de la transcriptasa reversa actúan como inductores del citocromo $\mathrm{P} 450$ produciendo una disminución de los niveles de inhibidores de la calcineurina9 Estas interacciones han de tenerse en cuenta al momento de ajustar el tratamiento inmunospresor y es aconsejable evitar el uso de inhibidores de la proteasa o inhibidores no nucleósidos de la transcriptasa reversa. En nuestro paciente decidimos mantener como tercera droga un inhibidor de la integrasa para evitar interacciones.

Respecto a la causa de la insuficiencia hepática se consideró la presencia de DILI. Se ha reportado hepatotoxicidad secundaria a abacavir/lamivudina $\left(\text { Kivexa }^{\circledR}\right)^{11}$, dolutegavir (Tivicay $\left.{ }^{\circledR}\right)^{12}$ y metformina $^{13}$. Existe en la literatura un reporte reciente de TH secundario a falla hepática subaguda por uso de dolutegavir ${ }^{14}$. No existen reportes de hepatotoxicidad grave secundaria a fentermina. No obstante, no es posible tener certeza de que la etiología de la insuficiencia hepática sea un DILI ni plantear cual sería la droga responsable por lo que restrospectivamente nos parece que la etiología debe considerarse criptogénica.

Inicialmente los resultados del $\mathrm{TH}$ en portadores de VIH previo al uso de TARV fueron desalentadores ${ }^{15,16}$. El registro de TH de la US United Network for Organ Sharing 18 identificó entre 1997 y 2006, 138 pacientes VIH+. La supervivencia global fue inferior en los receptores VIH+ $(n=138)$ en relación al grupo no VIH $(\mathrm{n}=30.520)$ a los $2 \mathrm{y}$ 3 años post-trasplante ( $70 \%$ y $60 \%$ versus $81 \%$ y $77 \%$ ), pero no existe registro de los cuidados perioperatorios del grupo portador de VIH por lo que desconocemos si fueron tratados de acuerdo a estándares actuales previo al trasplante. Un segundo estudio basado en el Scientific Registry of Transplant Recipients analizó 34.020 receptores, 180 de los cuales eran portadores de VIH. Este estudio separó su análisis en 2 períodos (20022007 y 2008-2011). En el primer período analizado los sujetos portadores de VIH presentaron un aumento de la mortalidad (hazard ratio ajustado o HRa 1,68, 95\% CI: 1,28-2,20, p < 0,001) y menor supervivencia del injerto (HRa 1,70, 95\% CI: 1,31-2,20, p < 0,001). Interesantemente, en el segundo período no se encontraron diferencias significativas en ninguno de los dos indicadores señalados (mortalidad HRa 1,11; 95\% CI: 0,52-
2,35, $\mathrm{p}=0,79$; supervivencia del injerto $\mathrm{HRa} 0,89$; 95\% CI: 0,42-1,88, $\mathrm{p}=0,77)$, lo que podría estar explicado por un mayor uso de TARV ${ }^{19}$. Respecto a aquellos pacientes co-infectados con virus de la hepatitis $\mathrm{C}$ es esperable que su pronóstico también mejore con el uso de antivirales de acción directa $^{19,20}$.

Gracias a la introducción de TARV, la infección por VIH puede considerarse una enfermedad crónica debido a la sustancial mejoría en su supervivencia. Dada su complejidad, los pacientes infectados por VIH con falla hepática aguda deben ser manejados por un equipo multidisciplinario en un centro de alto volumen de trasplantes.

El presente caso hace notar que el TH puede ser un procedimiento factible y exitoso toda vez que el paciente sea apropiadamente seleccionado y se tengan en cuenta las potenciales interacciones farmacológicas en el post trasplante.

\section{Referencias}

1. Kim WR, Lake JR, Smith JM, Skeans MA, Schladt DP, Edwards EB, et al. OPTN/SRTR 2015 Annual Data Report: Liver. Am J Transplant 2017; 17 Suppl 1: 174-251.

2. Roland ME, Stock PG. Liver transplantation in HIV-infected recipients. Semin Liver Dis 2006; 26 (3): 273-84.

3. Joshi D, O’Grady J, Taylor C, Heaton N, Agarwal K. Liver Transplantation in Human Immunodeficiency Virus-Positive Patients. Liver Transplant 2011; 17 (8): 881-90.

4. Cirioni O, Weimer LE, Fragola V, Giacometti A, Ancarani F, Maracci M, et al. A simplified HAART Regimen with Raltegravir and Lamivudine and pharmacokinetic interactions with a combined immunosuppressive therapy with tacrolymus and everolymus in an HIV/HCV/ HBV/HDV patient after Liver Transplantation. West Indian Med J 2014; 63 (7).

5. Clavien P. Acute liver failure: Where are the challenges? J Hepatol 2007; 46 (4): 553-4.

6. Sugawara Y, Tamura S, Kokudo N. Liver transplantation in HCV/HIV positive patients. World J Gastrointest Surg 2011; 3 (2): 21-8.

7. Morabito V, Grossi P, Lombardini L, Ricci A, Trapani S, Peritore D, et al. Solid Organ Transplantation in HIV+ Recipients: Italian Experience. Transplant Proc 2016; 48 (2): 424-30.

8. Norris S, Taylor C, Muiesan P, Portmann BC, Knisely AS, Bowles M, et al. Outcomes of liver transplantation in HIV-infected individuals: The impact of HCV and HBV infection. Liver Transplant 2004; 10 (10): 1271-78. 
9. Joshi D, Agarwal K. Role of liver transplantation in human immunodeficiency virus positive patients. World J Gastroenterol 2015; 21 (43): 12311-21.

10. Fung J, Eghtesad B, Patel-Tom K, DeVera M, Chapman $\mathrm{H}$, Ragni M. Liver transplantation in patients with HIV infection. Liver Transplant 2004; 10 (10): S39-S53.

11. Pezzani MD, Resnati C, Di Cristo V, Riva A, Gervasoni C. Abacavir-induced liver toxicity. Brazilian J Infect Dis 2016; 20 (5): 502-4.

12. https://www.gsksource.com/pharma/content/dam/glaxosmithkline/us/en/prescri bing_information/tivicay/ pdf/tivicay-pi-pil-.pdf

13. Olivera-González S, de Escalante-Yangüela B, Velilla-Soriano C, Amores-Arriaga B, Martín-Fortea P, Navarro-Aguilar ME. Hepatotoxicidad por metformina. Med Intensiva 2010; 34 (7): 483-7.

14. Wang B, Abbott L, Childs K, Taylor C, Agarwal K, Cormack I, et al. Dolutegravir-induced liver injury leading to sub-acute liver failure requiring transplantation: a case report and review of literature. Int J STD AIDS 2018; 29 (4): 414-7.

15. Tzakis A, Cooper M, Dummer J, Ragni M, Ward J, Starzl T. Transplantation in HIV+ patients. Transplantation
1990; 49 (2): 354-258.

16. Dummer JS, Erb S, Breinig MK, Ho M, Rinaldo Jr C, Gupta P, et al. Infection with human immunodeficiency virus in Pittsburgh transplant population. Transplantation 1989; 47 (1): 134-40.

17. Mindikoglu AL, Regev A, Magder LS. Impact of Human Immunodeficiency Virus on Survival After Liver Transplantation: Analysis of United Network for Organ Sharing Database. Transplantation 2008; 85 (3): 359-68.

18. Locke JE, Durand C, Reed RD, MacLennan P, Mehta S, Massie A, et al. Long-term Outcomes After Liver Transplantation Among Human Immunodeficiency Virus-Infected Recipients. Transplantation 2016; 100 (1): 141-6.

19. Campos-Varela I, Peters MG, Terrault NA. Advances in therapy for HIV/Hepatitis C virus-coinfected patients in the liver transplant setting. Clin Infect Dis 2015; 60 (1): 108-16.

20. Antonini TM, Furlan V, Teicher E, Haim-Boukovza S, Sebagh M, Coilly A, et al. Therapy with boceprevir or telaprevir in HIV/hepatitis $\mathrm{C}$ virus co-infected patients to treat recurrence of hepatitis $C$ virus infection after liver transplantation. AIDS 2015; 29 (1): 53-8. 